\title{
Double-blind comparison of ziprasidone and risperidone in the treatment of Chinese patients with acute exacerbation of schizophrenia
}

This article was published in the following Dove Press journal:

Neuropsychiatric Disease and Treatment

3 March 20I I

Number of times this article has been viewed

\author{
Hongyan Zhang' \\ Huafang $\mathrm{Li}^{2}$ \\ Liang Shu' \\ Niufan $\mathrm{Gu}^{2}$ \\ Gang Wang ${ }^{3}$ \\ Yongzhen Weng ${ }^{3}$ \\ Shiping $\mathrm{Xie}^{4}$ \\ Xinbao Zhang ${ }^{4}$ \\ Ting $\mathrm{Li}^{5}$ \\ Cui $\mathrm{Ma}^{5}$ \\ Wei Yu ${ }^{6}$ \\ Bruce Parsons ${ }^{7}$ \\ Manjula Schou ${ }^{8}$ \\ 'Institute of Mental Health, Peking \\ University, Beijing, China; ${ }^{2}$ Shanghai \\ Mental Health Center, Shanghai, \\ China; ${ }^{3}$ Capital Medical University, \\ Beijing An Ding Hospital, Beijing, \\ China; ${ }^{4}$ Nanjing Brain Hospital, \\ Nanjing, China; ${ }^{5}$ Guangzhou Brain \\ Hospital, Guangzhou, China; ${ }^{6}$ Pfizer \\ China, Beijing, China; ${ }^{7}$ Pfizer Inc, \\ New York, NY, USA; ${ }^{8}$ Pfizer Australia \\ Sydney, Australia
}

Background: The aim of the study was to evaluate the efficacy and safety of ziprasidone versus risperidone in Chinese subjects with acute exacerbation of schizophrenia.

Methods: In patients meeting the Chinese Classification of Mental Disorders criteria for schizophrenia and with a Positive and Negative Syndrome Scale (PANSS) total score $\geq 60$ were randomly assigned to six weeks of double-blind treatment with ziprasidone $40-80 \mathrm{mg}$ twice daily or risperidone $1-3 \mathrm{mg}$ bid, flexibly dosed. Noninferiority was demonstrated if the upper limit of the two-sided 95\% confidence interval (CI) for the difference in PANSS total score improvement from baseline in the evaluable population was smaller than the prespecified noninferiority margin of 10 units.

Results: The intent-to-treat population comprised 118 ziprasidone-treated and 121 risperidonetreated subjects. Improvement (reduction) from baseline to week 6 in PANSS total score was $(-35.6$ [95\% CI: $-38.6,-32.6])$ for ziprasidone and $(-37.1$ [95\% CI: $-39.9,-34.4])$ for risperidone. Noninferiority was demonstrated in the evaluable population with a difference score of 1.5 [95\% CI: $-2.5,5.5]$. Mean prolactin levels decreased at week 6 compared with baseline for ziprasidone $(-3.5 \mathrm{ng} / \mathrm{mL})$, but significantly increased for risperidone $(61.1 \mathrm{ng} / \mathrm{mL}$; $P<0.001)$. More risperidone-treated subjects (14.9\%) than ziprasidone-treated subjects $(4.2 \%)$ reported weight gain $\geq 7 \%$. Akathisia and somnolence in the ziprasidone group and akathisia and insomnia in the risperidone group were the most common side effects. Treatment-related/ treatment-emergent adverse events were reported by $79.7 \%$ and $71.1 \%$ of ziprasidone-treated and risperidone-treated subjects, respectively.

Conclusion: In Chinese subjects, ziprasidone was as effective as risperidone, with less weight gain and less prolactin elevation.

Keywords: ziprasidone, risperidone, schizophrenia

\section{Introduction}

Ziprasidone is a potent antagonist at 5-hydroxytriptamine (serotonin) 5-HT2A and dopamine D2 receptors, and has the highest ratio of affinities at these receptors among the available antipsychotic agents. ${ }^{1}$ In addition, ziprasidone is a potent 5-HT1A agonist, a potent 5-HT1D and 5-HT2C antagonist, and moderately inhibits 5-HT and noradrenaline reuptake sites in vitro. This unique collection of properties, in addition to its antipsychotic efficacy, may offer considerable potential benefits in treatment of the affective symptoms associated with schizophrenia, and also predicts a low propensity for extrapyramidal symptoms, sedation, and cognitive impairment. ${ }^{1}$ Ziprasidone has demonstrated a low propensity for drug interactions during in vitro and in vivo studies.

Several 4-6-week, fixed-dose (40-200 mg/day), placebo-controlled clinical trials in subjects with schizophrenia and schizoaffective disorder have demonstrated that
Correspondence: Hongyan Zhang Department of Psychiatry, Institute of Mental Health, Peking University, 5I North Huayuan Road, Beijing, I0019I, People's Republic of China Tel +861082801940 Fax +86 I0 820I3I83 Email sally_zhy@sina.com 
ziprasidone is an effective antipsychotic agent for positive, negative, and affective symptomatology associated with schizophrenia. ${ }^{2-4}$ The incidence and severity of adverse events associated with ziprasidone has been low. Ziprasidone demonstrates a relatively low incidence of extrapyramidal symptoms, and the incidence of postural hypotension and laboratory abnormalities has been found to be similar to that observed with placebo. ${ }^{2-4}$ A recent large, multinational, observational study comparing ziprasidone and olanzapine determined that, despite the known risk of QTc prolongation for ziprasidone, it was not associated with an elevated risk for nonsuicide-related (specifically cardiac) adverse events in real world use. ${ }^{5}$

Among the most extensively studied and widely used atypical agents, risperidone has been studied in patients with schizophrenia in comparison with haloperidol. ${ }^{6-10}$ Although risperidone has demonstrated efficacy in the treatment of schizophrenia, several studies have revealed that treatment with risperidone is accompanied by some tolerability problems, including extrapyramidal symptoms, serum prolactin elevation, and sexual dysfunction. ${ }^{11,12}$

The present study compared the efficacy, safety, and tolerability profiles of ziprasidone and risperidone in treating schizophrenia in Chinese subjects. It is among the first double-blind studies to compare two different atypical antipsychotic agents in Chinese subjects with schizophrenia. There have been few randomized, controlled clinical studies of atypical antipsychotics in Asian populations, and they have several important factors that vary between different racial and ethnic groups. ${ }^{13,14}$ Some studies have demonstrated differences in efficacious doses of antipsychotics, as well as antipsychotic response, among different racial and ethnic groups. ${ }^{13,15,16}$ However, some other studies have failed to replicate these findings. ${ }^{17,18}$ Hence, the additional aim of this study was to document differences, if any, in average dose, clinical response, and safety profile of ziprasidone between Chinese and Western populations.

\section{Methods}

\section{Subjects}

The trial included male and female inpatients aged 18-65 years. At randomization, subjects with a diagnosis of schizophrenia according to the Chinese Classification of Mental Disorders (CCMD-3) criteria were hospitalized, and were required to have a Positive and Negative Syndrome Scale (PANSS) score $\geq 60 .{ }^{19}$ Written informed consent was obtained from all subjects. Exclusion criteria included a CCMD-3-defined diagnosis of substance abuse within the three months prior to randomization or any of the following prior to randomization: intermittent (within 12 hours) use or planned regular use (within one week) of antipsychotic agents, depot agents (the longer of two weeks or one cycle), treatment with antidepressants (one week, except two weeks for monoamine oxidase inhibitors including meclobemide and five weeks for fluoxetine), mood stabilizers (one week), regular administration of clozapine (within three months), or regular administration of risperidone (within four weeks). Exclusion criteria also included receiving another investigational agent one month prior to screening, termination of risperidone due to nonresponse or intolerability, resistance to conventional antipsychotics, at immediate risk of the impulse to commit harm to self or others, QTc prolongation or a predrug QTc of $\geq 450 \mathrm{msec}$, pregnant or lactating women, or women of childbearing potential not using an acceptable method of contraception, confirmed clinically significant abnormal laboratory values and/or electrocardiogram as determined by a general physician or cardiologist.

\section{Study design}

This was a randomized, double-blind, double-dummy, activecontrolled, parallel-group, multicenter study. The Chinese State Food and Drug Administration required that a minimum of 100 pairs of subjects complete the study. Therefore, this study aimed to enroll 240 subjects. The additional subject numbers allowed for approximately $17 \%$ nonevaluability. At baseline, subjects received double-dummy study drug (ziprasidone capsules or risperidone capsules, and matching placebo). Subjects received all study drugs with food and under supervision. During the first week, the dose was steadily titrated upwards for all subjects (see Table 1 for dosing regimen). At the end of week 1, the dosage was titrated up or down, based on the clinical status of the subject and the safety and tolerability of the study drug. Any medication taken, other than the study drug, was considered to be concomitant medication. All concomitant medications taken during the three months prior to screening and during the course of the study were recorded. Specifically, standard anticholinergics were permitted when deemed necessary, but not prophylactically, while benzodiazepines could be administered parenterally for the first three days of the

Table I Study drug regimen

\begin{tabular}{lll}
\hline Day/week & Ziprasidone, $\mathbf{m g} /$ day $^{\mathbf{a}}$ & Risperidone, $\mathbf{~ m g / d a y}^{\mathbf{b}}$ \\
\hline Day I-2 & 80 & $\mathrm{I}$ \\
Day 3-4 & 120 & 2 \\
Day 5-7 & 120 & 3 \\
Week 2-6 & 80,120 , or 160 & 2,4 , or 6
\end{tabular}

Notes: ${ }^{2} Z$ iprasidone was dosed twice daily; ${ }^{\circ}$ Risperidone was dosed once daily for the first seven days, then twice daily thereafter. 
study (clonazepam up to $4 \mathrm{mg}$ or surazepam up to $2 \mathrm{mg}$ per night). Standard procedures were used to ensure rater training and reliability during the investigation. A double-dummy method was used to keep both subjects and investigators blinded to the treatment allocation. A computer-generated randomization schedule was used to randomize subjects to either ziprasidone or risperidone, and was accessed by the investigators via an interactive voice response system each time a eligible subject was available for randomization.

The study was conducted in compliance with the Declaration of Helsinki and the International Electrotechnical Commission, informed consent regulations, and International Conference on Harmonization, and Good Clinical Practices guidelines. In addition, all local regulatory requirements were followed, in particular, those affording greater protection to the safety of trial participants.

\section{Evaluation of efficacy and safety}

The primary efficacy evaluation was the PANSS total score. Secondary efficacy evaluations included the Brief Psychiatric Rating Scale (BPRSd [derived from 18 PANSS items]), PANSS subscales (positive, negative, general psychopathology) and responder rate (defined as $\geq 50 \%$ reduction from baseline PANSS total score), and Clinical Global Impression-Severity (CGI-S) and CGI-Improvement (CGI-I) scales.

All observed or self-reported adverse events, regardless of treatment group or suspected causal relationship to the study drugs, were recorded. The severity, duration, and possible relationship to the study drugs of all adverse events were also recorded. Laboratory tests, performed at screening and the end of treatment, included aspartate aminotransferase, alanine aminotransferase, alkaline phosphatase, blood glucose (eight-hour fasting), and serum prolactin. The rating scale for extrapyramidal side effects (known as the Simpson-Angus scale), and Barnes Akathisia scale were also measured. ${ }^{20}$ Physical examination findings, vital signs, and electrocardiographic parameters, including QTc intervals (using the Bazett correction), were recorded.

\section{Statistical analysis}

This study included a safety population and two efficacy analysis populations, ie, the intent-to-treat population and the evaluable population. All efficacy variables were analyzed using the intent-to-treat population. Only the primary measure was analyzed using the evaluable population. The intent-to-treat population was defined as any subject who took at least one dose of study medication and had a baseline and a follow-up (postbaseline) efficacy measurement. The evaluable population was a subset of the intent-to-treat population, and comprised subjects with a baseline and end of study (week 6) PANSS total score, no major protocol deviations, and compliance with study medication $(>70 \%$ or $<120 \%$ ). The safety population consisted of all subjects who took at least one dose of study medication.

Detailed descriptive summaries of efficacy and safety outcomes are tabulated, along with summaries of adverse events, treatment discontinuations, vital signs, laboratory assessments, electrocardiographic parameters, physical examinations, concomitant medications, and treatments for the safety population.

All tests of hypotheses were two-sided and conducted at the 5\% significance level without adjustment for multiple comparisons, and all confidence intervals (CIs) were two-sided at the $95 \%$ level or suitable data transformations applied, and nonparametric methods were used. If the response at end of study was missing for the primary efficacy endpoint (intent-to-treat population analysis), secondary efficacy or safety endpoints (Simpson-Angus scale and Barnes Akathisia scale), or the last available postbaseline observation was carried forward (LOCF).

The primary endpoint was change in the PANSS total score from baseline to study end. Assuming a two-sided significance level of $5 \%$, the same underlying mean change from baseline in PANSS total score for the two treatments, a common standard deviation (SD) of 25 units, and a nonevaluability rate of $17 \%$, a sample size of 240 subjects allowed for the noninferiority of ziprasidone compared with risperidone to be established within 10 units (noninferiority margin) with $80 \%$ power.

Change in PANSS positive subscale score from baseline to end of study, change in PANSS negative subscale score from baseline to end of study, change in PANSS general psychopathology score from baseline to end of study, and change in BPRSd score from baseline to end of study were analyzed using an analysis of covariance (ANCOVA) model with treatment group and study center as factors, and the baseline score as a covariate. The least squares mean, standard error, $95 \% \mathrm{CI}$ for the mean difference, and $P$ value were presented for all endpoints analyzed using ANCOVA. Responder rate, as derived from the reduction from baseline in the PANSS total score at end of study, was analyzed using the Cochran-Mantel-Haenszel test stratified by study center. An estimate of the overall odds ratio (stratified by study center) and its associated $95 \%$ CI were also presented.

CGI-S and CGI-I scores at end of study were analyzed using Wilcoxon rank-sum tests stratified by study center. Assessment of the difference between the treatment groups with respect to the proportion of subjects achieving a 
score $\leq 2$ versus $>2$ at the end of study was also carried out (Cochran-Mantel-Haenszel test stratified by study center).

Median changes in prolactin values from baseline to LOCF were summarized. Descriptive summaries of the SimpsonAngus scale and the Barnes Akathisia scale were presented. Changes from baseline to end of study across treatment groups comparing the Simpson-Angus scale total score and the global clinical assessment of akathisia based on the Barnes Akathisia scale (Cochran-Mantel-Haenszel stratified by center) used the ANCOVA (with treatment group and study center as factors, and the baseline score as a covariate). Changes from screening to end of study in serum prolactin were analyzed using ANCOVA with treatment, study center, and gender of subject fitted as factors, and screening serum prolactin value as a covariate. Physical examination findings, vital signs, electrocardiographic parameters, including QTc intervals, were reviewed for all patients.

\section{Results}

\section{Demographic and clinical characteristics of subjects}

In total, 253 subjects were screened for entry into this study, 242 were randomly assigned to treatment and 239 received treatment (Table 2). One hundred eighteen subjects and 121 subjects were assigned to receive ziprasidone and risperidone, respectively, and 97 (82\%) and 111 (92\%) completed the study, respectively. The subjects' demographic and baseline clinical characteristics are summarized in Table 3. All subjects were Asian, with a primary diagnosis of schizophrenia (mean duration, range: 6.4 years, $0.0-38.0$ years and 5.3 years, $0.0-36.6$ years for the ziprasidone and risperidone groups, respectively). Treatment groups were comparable for all baseline characteristics.

Table 2 Subject disposition

\begin{tabular}{|c|c|c|}
\hline Screened & $n=253$ & \\
\hline \multirow[t]{2}{*}{ Assigned to treatment } & \multicolumn{2}{|l|}{$n=242$} \\
\hline & Ziprasidone & Risperidone \\
\hline Treated, n (\%) & $118(100)$ & $121(100)$ \\
\hline Completed, n (\%) & $97(82)$ & III (92) \\
\hline Discontinued, n (\%) & $21(18)$ & $10(8)$ \\
\hline \multicolumn{3}{|l|}{ Analyzed for efficacy, n (\%) } \\
\hline ITT population & $118(100)$ & $121(100)$ \\
\hline Evaluable population & $96(8 I)$ & III (92) \\
\hline Discontinuations, $\mathrm{n}$ & 21 & 10 \\
\hline Related to study drug & 18 & 7 \\
\hline Adverse events & 8 & 2 \\
\hline Lack of efficacy & 9 & 5 \\
\hline Laboratory abnormality & I & 0 \\
\hline Unrelated to study drug & 3 & 3 \\
\hline
\end{tabular}

Abbreviation: ITT, intent-to-treat.
Table 3 Baseline subject demographic and clinical characteristics

\begin{tabular}{lll}
\hline & $\begin{array}{l}\text { Ziprasidone } \\
\text { (n= I I 8) }\end{array}$ & $\begin{array}{l}\text { Risperidone } \\
\text { (n= I I I) }\end{array}$ \\
\hline Gender, female, $\mathrm{n}(\%)$ & $58(49)$ & $62(5 \mathrm{I})$ \\
Age, years, mean (SD) & $34.7(10.8)$ & $34.8(10.9)$ \\
Weight, kg, mean (SD) & $63.5(12.1)$ & $61.2(10.1)$ \\
Height, cm, mean (SD) & $165.4(7.2)$ & $165.9(7.6)$ \\
Baseline PANSS score & & \\
$\quad$ Total & $84.4(13.6)$ & $84.6(13.2)$ \\
Positive & $23.8(5.5)$ & $23.3(5.8)$ \\
$\quad$ Negative & $20.8(7.6)$ & $21.5(6.8)$ \\
$\quad$ General psychopathology & $39.8(7.6)$ & $39.8(8.3)$ \\
Baseline BPRSd score & $46.5(7.8)$ & $45.8(7.5)$ \\
\hline
\end{tabular}

Abbreviations: SD, standard deviation; PANSS, Positive and Negative Syndrome Scale; BPRSd, Brief Psychiatric Rating Scale derived from 18 PANSS items.

\section{Dosing}

This was a flexible-dose study in which the investigators were allowed to assign doses according to clinical judgment within the permissible range. At the end of treatment or at early termination, the proportion of subjects on the highest dose was comparable between the groups, ie, $21 \%$ for ziprasidone and $23 \%$ for risperidone. However, the proportion of subjects receiving the low and medium doses was different between treatment groups: low dose, $17 \%$ ziprasidone; $5 \%$ risperidone: medium dose, $62 \%$ ziprasidone; $72 \%$ risperidone. The mean daily prescribed dose by week is detailed in Table 4 . The mean dosage received in the ziprasidone and risperidone groups was $118.5 \mathrm{mg}(\mathrm{SD}=18.1)$ and $3.8 \mathrm{mg}(\mathrm{SD}=0.8)$, respectively.

Eighty-two (69.5\%) ziprasidone-treated and 79 (65.3\%) risperidone-treated subjects received clonazepam during the study. Sixty-two (52.5\%) ziprasidone-treated and 56 (46.3\%) risperidone-treated subjects received trihexyphenidyl during the study. While the study protocol permitted anticholinergic administration only as deemed clinically necessary, an earlier study had documented the need for prophylactic antiparkinsonian medication concomitant with neuroleptics to manage severe extrapyramidal symptoms. ${ }^{21}$

\section{Efficacy}

Primary efficacy endpoint

The extent of improvement (reduction) from baseline in PANSS total score was statistically significant and comparable

Table 4 Prescribed daily dose by week

\begin{tabular}{|c|c|c|c|c|}
\hline \multirow[t]{2}{*}{ Treatment } & \multicolumn{4}{|l|}{ Week } \\
\hline & $I$ & 2 & 4 & Last visit \\
\hline $\begin{array}{l}\text { Ziprasidone, } \\
\text { mean (n) }\end{array}$ & $116.4(110)$ & $122.3(106)$ & $122.0(100)$ & $121.7(118)$ \\
\hline $\begin{array}{l}\text { Risperidone, } \\
\text { mean (n) }\end{array}$ & $3.8(1 \mid 4)$ & $4.2(114)$ & $4.3(I \mid 3)$ & $4.4(12 I)$ \\
\hline
\end{tabular}


for both treatment groups (Figure 1). At week 6, the difference between treatment groups was found to be 1.5 (standard error [SE] 2.03; 95\% CI: -2.5, 5.5). Ziprasidone was found to be noninferior to risperidone at week 6 for the evaluable population; improvement from baseline to week 6 in PANSS total score was $-35.6(95 \% \mathrm{CI}:-38.6,-32.6)$ for ziprasidone and -37.1 (95\% CI: $-39.9,-34.4)$ for risperidone. The upper limit of the $95 \%$ CI was well within the specified 10 units. Noninferiority of ziprasidone over risperidone was confirmed by the intent-to-treat population results, ie, least squares mean 4.4 (SE 2.46, 95\% CI: $-0.4,9.3$ ).

\section{Secondary efficacy endpoints}

Statistically significant changes from baseline were observed for both treatment groups on all PANSS subscales and BPRSd (Table 5). No statistically significant differences between ziprasidone and risperidone were observed, apart from the PANSS general psychopathology score. This difference was not supported when analyzed using rank generalized linear models, consistent with the non-normality observed for this endpoint $(P=0.081)$.

The responder rate increased over time and was comparable between treatment groups at each time point. At the LOCF endpoint, 76 (64.4\%) ziprasidone-treated and 87 (71.9\%) risperidone-treated subjects had achieved a response. The odds of achieving a response to treatment with ziprasidone were similar to achieving a response to risperidone (odds ratio 0.71; 95\% CI: $0.41,1.24)$.

Scores on CGI-I and CGI-S were improved for both treatment groups. However, a significantly greater improvement was observed in the risperidone group on the CGI-I scale at week 6 compared with the ziprasidone group (mean at week 6: risperidone 2.2 [SD 1.15], ziprasidone 2.6 [SD 1.31], $P=0.039$ ), with $66.1 \%$ of risperidone-treated subjects

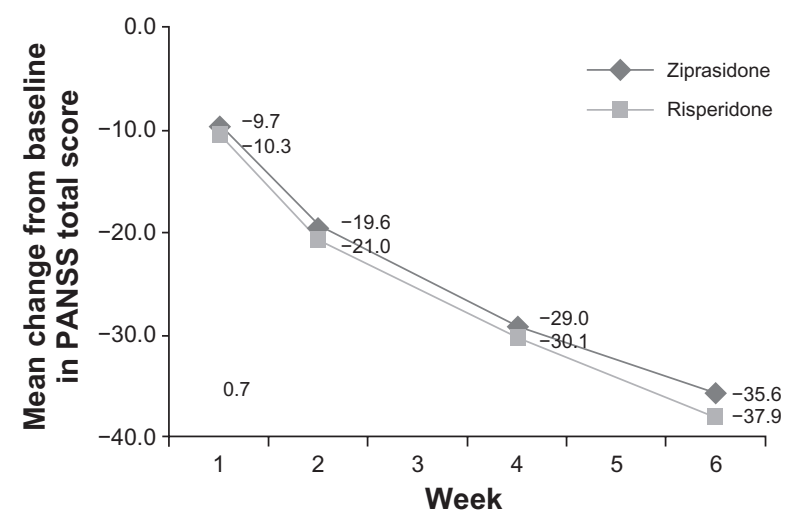

Figure I Mean change from baseline Positive and Negative Syndrome Scale total score (evaluable population).

Abbreviation: PANSS, Positive and Negative Syndrome Scale.
Table 5 LS mean change in PANSS subscales and BPRSd scores (ITT population)

\begin{tabular}{|c|c|c|c|}
\hline & \multicolumn{3}{|c|}{ Change from baseline to week 6 (LOCF) } \\
\hline & LS mean (SE) & 95\% Cl & $P$ value \\
\hline \multicolumn{4}{|l|}{ PANSS positive } \\
\hline Ziprasidone & $-10.2(0.58)$ & -11.3 to -9.0 & $<0.001$ \\
\hline Risperidone & $-11.5(0.57)$ & -12.6 to -10.3 & $<0.001$ \\
\hline $\begin{array}{l}\text { Difference between } \\
\text { treatment groups }\end{array}$ & $1.3(0.80)$ & -0.3 to 2.9 & 0.105 \\
\hline \multicolumn{4}{|l|}{ PANSS negative } \\
\hline Ziprasidone & $-7.0(0.53)$ & -8.1 to -6.0 & $<0.001$ \\
\hline Risperidone & $-7.8(0.52)$ & -8.8 to -6.8 & $<0.001$ \\
\hline $\begin{array}{l}\text { Difference between } \\
\text { treatment groups }\end{array}$ & $0.8(0.73)$ & -0.7 to 2.2 & 0.292 \\
\hline \multicolumn{4}{|l|}{$\begin{array}{l}\text { PANSS general } \\
\text { psychopathology }\end{array}$} \\
\hline Ziprasidone & $-12.3(0.79)$ & -13.8 to -10.7 & $<0.001$ \\
\hline Risperidone & $-14.7(0.78)$ & -16.2 to -13.2 & $<0.001$ \\
\hline $\begin{array}{l}\text { Difference between } \\
\text { treatment groups }\end{array}$ & $2.4(1.09)$ & 0.3 to 4.6 & 0.027 \\
\hline \multicolumn{4}{|l|}{ BPRSd } \\
\hline Ziprasidone & $-16.1(0.98)$ & -18.0 to -14.1 & $<0.001$ \\
\hline Risperidone & $-18.4(0.97)$ & -20.3 to -16.5 & $<0.001$ \\
\hline $\begin{array}{l}\text { Difference between } \\
\text { treatment groups }\end{array}$ & $2.3(1.36)$ & -0.4 to 5.0 & 0.088 \\
\hline
\end{tabular}

Abbreviations: LS, least squares; PANSS, Positive and Negative Syndrome Scale BPRSd, Brief Psychiatric Rating Scale derived from I8 PANSS items; ITT, intent-totreat; LOCF, last observation carried forward; $\mathrm{Cl}$, confidence interval.

achieving a CGI-I score $\leq 2$ at week 6 compared with $55.9 \%$ in the ziprasidone group $(P=0.115)$.

\section{Safety and tolerability \\ Weight gain}

Risperidone was associated with significant weight gain ( $\geq 7 \%$ ) in 18 (14.9\%, 95\% CI: 9.06, 22.49) subjects, whereas only five $(4.2 \%, 95 \%$ CI: $1.39,9.61)$ ziprasidone subjects had significant weight gain at the end of the study compared

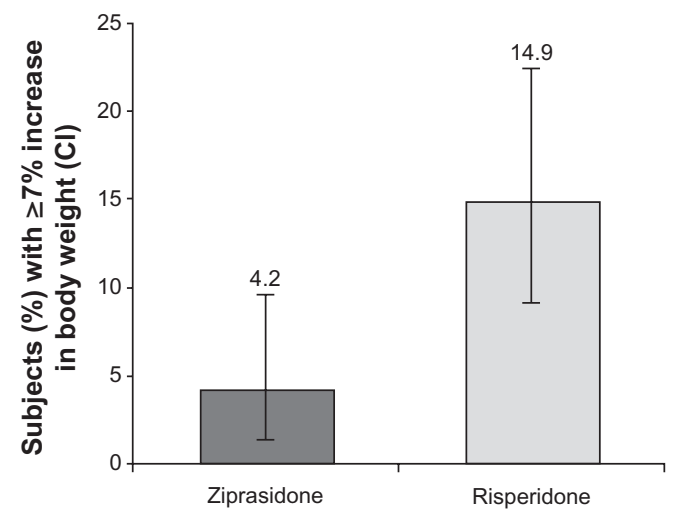

Figure 2 Proportion of subjects with a $\geq 7 \%$ gain in body weight from baseline (safety population).

Abbreviation: $\mathrm{Cl}$, confidence interval. 
with baseline (Figure 2). No change was observed in median fasting glucose levels from baseline to last observation for both ziprasidone and risperidone groups ( $5 \mathrm{mg} / \mathrm{dL})$.

\section{Serum prolactin}

While serum prolactin levels increased from screening to week 6 in the risperidone group, mean prolactin levels decreased slightly in the ziprasidone group (Figure 3). A greater proportion of subjects in the risperidone group had significant prolactin abnormalities compared to the ziprasidone group. Among men treated with risperidone, all 16 subjects $(100 \%)$ who were normal at baseline and 28 of $29(96.6 \%)$ subjects who were abnormal at baseline were reported to have abnormal serum prolactin levels. The corresponding values for ziprasidone-treated men were nine of 21 (42.9\%) and 15 of 25 (60.0\%), respectively. Among women treated with risperidone, the corresponding values were 13 of $13(100 \%)$ and 38 of $39(97.4 \%)$, respectively, compared with 9 of $15(60.0 \%)$ and 23 of $37(62.2 \%)$, respectively for ziprasidone-treated women.

\section{Movement disorders}

Ziprasidone and risperidone were comparable for change from baseline in both movement disorder scales. At week 6, Simpson-Angus scale scores were slightly increased compared with baseline in both groups. The least squares mean change from baseline was 0.9 (95\% CI: $0.4,1.4)$ in the ziprasidone group and $0.5(95 \% \mathrm{CI}: 0.0,1.0)$ in the risperidone group (treatment difference $0.4,95 \% \mathrm{CI}:-0.3,1.1$ ). The majority of subjects did not experience worsening of symptoms (compared with baseline) on the Barnes Akathisia scale, and $83 \%$ and $88 \%$ of subjects in the ziprasidone and risperidone

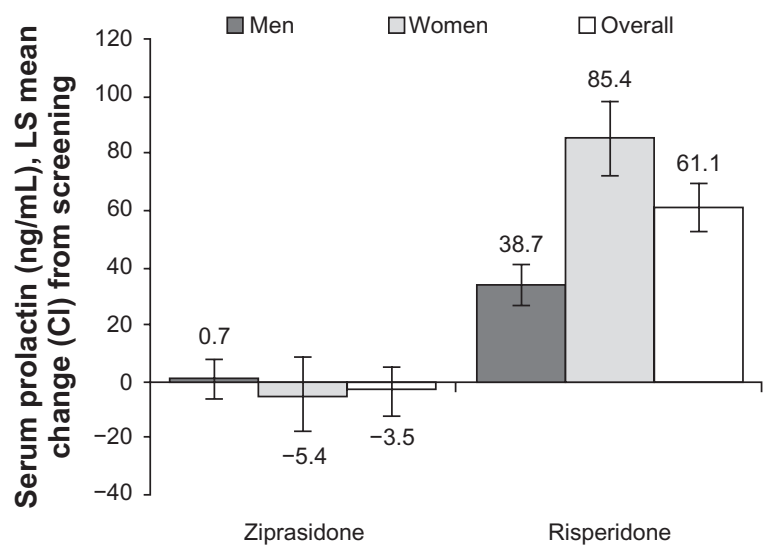

Figure 3 Mean change in serum prolactin from screening to week 6 (safety population).

Abbreviations: $\mathrm{Cl}$, confidence interval; $\mathrm{LS}$, least squares. groups, respectively, improved or experienced no change in symptoms.

\section{Adverse events}

In total, 94 (79.7\%) ziprasidone-treated subjects and 86 (71.1\%) risperidone-treated subjects experienced 198 and 152 treatment-related adverse events, respectively. The body system most affected by study treatment was the nervous system, with $70(59 \%)$ and 51 (42\%) subjects reporting adverse events in the ziprasidone and risperidone groups, respectively. Treatment-related adverse events ( $\geq 5 \%$ in any treatment group) are summarized in Table 6. The majority of adverse events were mild or moderate in severity. Three adverse events in each group were reported as severe: the ziprasidone group reported severe akathisia $(n=43$, $36.4 \%$ ), neuroleptic malignant syndrome $(n=1,0.9 \%)$, and somnolence $(\mathrm{n}=11,9.3 \%)$; the risperidone group reported severe akathisia $(n=2)$ and insomnia $(n=1)$. Eight subjects $(6.8 \%)$ in the ziprasidone group and two subjects $(1.7 \%)$ in the risperidone group withdrew permanently from the study due to treatment-related adverse events. One additional subject discontinued from the ziprasidone group due to abnormal laboratory test results. This subject had normal baseline alanine aminotransferase (21 U/L) and aspartate aminotransferase (31 U/L) levels, but alanine aminotransferase (115 U/L) and aspartate aminotransferase (57 U/L) increased on day 29.

One subject had a serious adverse event during treatment with ziprasidone. The physician reported that the subject suffered from severe neuroleptic malignant syndrome characterized by fever $\left(39.4^{\circ} \mathrm{C}\right)$ and significant extrapyramidal symptoms, along with the consciousness disorder. Treatment

Table 6 Treatment-related, treatment-emergent adverse events occurring in $\geq 5 \%$ of subjects

\begin{tabular}{lll}
\hline & $\begin{array}{l}\text { Ziprasidone } \\
(\mathbf{n}=\mathbf{I} \text { I8) } \\
\mathbf{n}(\%)\end{array}$ & $\begin{array}{l}\text { Risperidone } \\
(\mathbf{n}=\mathbf{I} \text { | I }) \\
\mathbf{n}(\%)\end{array}$ \\
\hline Akathisia & $43(36.4)$ & $29(24.0)$ \\
Extrapyramidal disorder & $26(22.0)$ & $20(16.5)$ \\
Constipation & $17(14.4)$ & $19(15.7)$ \\
Somnolence & $11(9.3)$ & $2(1.7)$ \\
Dystonia & $8(6.8)$ & $6(5.0)$ \\
ALT increased & $7(5.9)$ & $14(11.6)$ \\
AST increased & $7(5.9)$ & $6(5.0)$ \\
Dizziness & $6(5.1)$ & $8(6.6)$ \\
Palpitations & $6(5.1)$ & $6(5.0)$ \\
Sinus tachycardia & $6(5.1)$ & $2(1.7)$ \\
\hline
\end{tabular}

Abbreviations: ALT, alanine aminotransferase; AST, aspartate aminotransferase. 
was halted, and the subject recovered after being permanently discontinued from the study, which suggested that this adverse event was induced by ziprasidone.

\section{Vital signs}

Slight decreases in resting pulse rate were observed (ziprasidone, -1.00 beats/minute; risperidone, -4.00 beats/ minute). In both treatment groups, the median measured resting systolic and diastolic blood pressure was $120 \mathrm{mmHg}$ and $75 \mathrm{mmHg}$, respectively.

\section{Electrocardiographic parameters}

The majority of subjects ( $\mathrm{n}=117$ in both treatment groups) had an electrocardiogram performed at screening and had electrocardiographic results within normal limits (ziprasidone $\mathrm{n}=89$ and risperidone $\mathrm{n}=97$ ). The mean (SD) values of QTc (Bazett correction) at baseline and last observation for the ziprasidone group were 403.6 (24.52) $\mathrm{msec}$ and 403.6 (32.26) $\mathrm{msec}$ and for the risperidone group were 402.3 (26.71) msec, and 399.9 (25.21) msec, respectively. Accordingly, the QTc change (SD) from baseline to last observation for ziprasidone and risperidone was 0.00 (33.1) sec and -2.4 (27.8) msec, respectively. One subject on ziprasidone had an increased QTc interval postbaseline from $360 \mathrm{msec}$ at baseline to $511 \mathrm{msec}$, but showed no corresponding clinical manifestations.

\section{Discussion}

In this Chinese sample, ziprasidone was determined to be as effective as (noninferior to) risperidone, as determined by the primary efficacy measure, ie, change in PANSS total score from baseline to week 6. Similar improvements from baseline to week 6 on PANSS positive and negative subscales, BPRSd, PANSS responder rate, and CGI-S, were observed for ziprasidone and risperidone. The statistically significant treatment difference in the PANSS general psychopathology subscale was not supported when the data were analyzed using a prespecified rank generalized linear model. However, a statistically significant treatment difference in favor of risperidone was observed for the CGI-I at week 6. Furthermore, ziprasidone demonstrated a low propensity for weight gain and had neutral effects on prolactin levels, compared with risperidone.

One ziprasidone-treated subject had a QTc value of $\geq 500 \mathrm{msec}$, but showed no corresponding clinical manifestations. QTc prolongation in ziprasidone-treated subjects occurs rarely. The safety profile of ziprasidone indicates that, as of February 2000, 2 of 3095 (0.064\%) subjects receiving ziprasidone demonstrated QTc values $\geq 500$ msec compared with one of 440 (2.3\%) subjects receiving placebo. ${ }^{22}$ Furthermore, of the ziprasidone-treated subjects in the safety database, neither case suggested a role for ziprasidone.

Because the majority of clinical studies of atypical antipsychotics have been conducted in largely Western populations, randomized, double-blind studies of atypical antipsychotics in Asian populations are rare. In a 12-week, randomized, double-blind study in Chinese inpatients, treatment with risperidone resulted in a significantly greater reduction in PANSS total score compared with haloperidol, with fewer side effects. ${ }^{23}$ While baseline PANSS total scores in the present study (ziprasidone group, 84.4; risperidone group, 84.6) were similar to those for the aforementioned study (risperidone group, 82.4; haloperidol group, 79.3), a greater reduction in PANSS total score was observed in the present study for risperidone-treated subjects. In a 24-week, randomized, double-blind, multicenter study of olanzapine versus haloperidol in Asian outpatients, subjects treated with both medications improved significantly within eight weeks. ${ }^{24}$ Changes in PANSS total scores from baseline to week 8 are similar to the changes observed in the present study. In a four-week, double-blind, randomized, parallel study of aripiprazole and risperidone in Chinese inpatients, both treatment groups demonstrated improvement from baseline in PANSS total, PANSS positive, and PANSS negative scores, as well as CGI-S scores at the study endpoint. ${ }^{25}$ Baseline scores were similar to the present study, and changes from baseline PANSS scores to week 4 were comparable (change from baseline to week 4 [intent-to-treat]: present study, ziprasidone, -16.6 ; risperidone, -19.7 versus Chan et $\mathrm{al}^{25}$ aripiprazole -19.6 ; risperidone, -21.1). In addition to the comparable efficacy of ziprasidone and risperidone observed in the present study, another comparative study of ziprasidone and risperidone in combination with adjunctive clozapine for schizophrenia also showed similar efficacy for these two atypical antipsychotics. ${ }^{26,27}$

Baseline psychopathology scores in the present study (PANSS: ziprasidone, 84.4, risperidone, 84.6; BPRS: ziprasidone, 46.5, risperidone 45.8) appear to be slightly lower than scores reported for short-term Western studies of ziprasidone in subjects with schizophrenia. Future studies will be required to determine whether the severity of psychopathology at baseline is consistently lower in Chinese populations, or if this finding is unique to this study. Daniel et al reported mean baseline PANSS total scores between 
95.8 and 98.2, PANSS negative subscale scores between 24.3 and 25.4, and BPRS total scores between 55.0 and 56.5. ${ }^{3}$ Simpson et al reported mean baseline PANSS total scores, negative subscale scores, and positive subscale scores of 90 , 22.2, and 23.3, respectively, and BPRS total score of 51.5. ${ }^{28}$ Keck et al reported mean baseline BPRS total scores ranging between 36.5 and 37.0. ${ }^{2}$ Addington et al reported mean baseline PANSS total scores of 93.8 and 97.6 for ziprasidone and risperidone groups, respectively. ${ }^{29}$ It should be noted that baseline psychopathology scores in CATIE (Clinical Antipsychotic Trials of Intervention Effectiveness) were lower, ranging from 74.3 to 76.4. However, these results may not be comparable due to differences in study design.

Of the aforementioned studies, the design of the Addington et al study, an eight-week, double-blind, randomized, multicenter comparison of ziprasidone and risperidone in the treatment of acute exacerbation of schizophrenia or schizoaffective disorder, is most similar to the present study. ${ }^{29}$ While baseline PANSS total scores in the Addington study were higher than in our study, changes from baseline to study endpoint were smaller (in the Addington et al study, ziprasidone, -25.8; risperidone, -27.3). Responder rates, as defined by a $\geq 50 \%$ reduction from baseline PANSS total score, were higher in the present study. The total mean prescribed daily dose of ziprasidone was similar between studies; the total mean prescribed dose of risperidone was higher in the Addington study $(7.39 \mathrm{mg} /$ day overall versus $3.7 \mathrm{mg}$ /day in the present study). However, this study has a few limitations, including the relatively short observational period, absence of a placebo group, and lack of scales evaluating depressive symptoms.

In our study, ziprasidone had neutral effects on mean prolactin levels, but the use of risperidone was associated with significant increases in mean prolactin levels from baseline in both men and women. The low rates of prolactin elevation with ziprasidone treatment compared with other atypicals is thought to be due to the mechanism of action of the drug (offsetting effects on 5-HT1A and 5-HT2A receptors). ${ }^{30}$ Clinically significant elevations in prolactin were more common in risperidone-treated subjects than in ziprasidone-treated subjects in the Addington et al study as well.

The baseline weight of subjects in the present study is considerably lower than baseline weights reported in Western studies. Here, baseline weights in the ziprasidone and risperidone groups were $63.5 \mathrm{~kg}$ and $61.2 \mathrm{~kg}$, respectively. Mean baseline weights in several short-term Western trials of ziprasidone in schizophrenia ranged from $77.9 \mathrm{~kg}$ to
$87.1 \mathrm{~kg}$ in men, and from 64.5 to $80.7 \mathrm{~kg}$ in women. ${ }^{2,3,28}$ A difference in height may partially account for differences in baseline weight between this study population and Western study populations; in the Simpson study, ${ }^{28}$ mean height was $171.9 \mathrm{~cm}$ versus $165.4 \mathrm{~cm}$ and $165.9 \mathrm{~cm}$ for ziprasidone and risperidone, respectively, in the present study. Despite these baseline differences in weight between the present study population and the Western study populations, the incidence of weight gain $\geq 7 \%$ of baseline body weight in the present study was similar to Western studies (present study, $15 \%$ of risperidone-treated subjects versus $4 \%$ of ziprasidone-treated subjects; Addington et al study, 16.0\% of risperidone-treated subjects versus $8.2 \%$ of ziprasidonetreated subjects; CATIE, $14.0 \%$ of risperidone-treated subjects versus $4.0 \%$ of ziprasidone-treated subjects). ${ }^{29,31}$ Other metabolic parameters, such as lipid metabolism and heart rate variability, were not measured in this study, although resting blood pressure and fasting glucose levels were similar between the treatment groups. Rates of movement disorders, as measured by the Barnes Akathisia scale, were similar between the studies.

In this Chinese sample, we found that ziprasidone was as effective (noninferior) as risperidone for treating acute exacerbation of schizophrenia, as determined by the PANSS total score. The safety profiles of these two drugs were comparable. Overall, ziprasidone was safe and well tolerated in this Chinese population. Few randomized placebo-controlled studies of atypical antipsychotics in Chinese subjects with schizophrenia exist, because the majority of studies are conducted in Western populations. The Chinese subjects in the present study had slightly lower baseline psychopathology scores than what has been reported for several Western studies, but experienced substantial improvement following treatment. Chinese subjects had lower weight at baseline than subjects in Western studies. Despite baseline differences, Chinese subjects treated with risperidone experienced weight gain and prolactin elevation, as is often seen in Western studies. By contrast, ziprasidone demonstrated lower liability for weight gain and favorable effects on prolactin levels. Here, we have established that ziprasidone is an appropriate treatment option for Chinese subjects with schizophrenia.

\section{Disclosure}

This study was sponsored by Pfizer Inc. MS worked for Pfizer during the study duration and manuscript preparation, but is no longer employed by Pfizer. Editorial Support was provided by Annie Neild, PhD, of PAREXEL and was funded 
by Pfizer Inc. Otherwise the investigators report no conflicts of interest in this work.

\section{References}

1. Seeger TF, Seymour PA, Schmidt AW, et al. Ziprasidone (CP-88,059): A new antipsychotic with combined dopamine and serotonin receptor antagonist activity. J Pharmacol Exp Ther. 1995;275(1):101-113.

2. Keck P Jr, Buffenstein A, Ferguson J, et al. Ziprasidone 40 and $120 \mathrm{mg} /$ day in the acute exacerbation of schizophrenia and schizoaffective disorder: A 4-week placebo-controlled trial. Psychopharmacology (Berl). 1998;140(2):173-184.

3. Daniel DG, Zimbroff DL, Potkin SG, Reeves KR, Harrigan EP, Lakshminarayanan M. Ziprasidone $80 \mathrm{mg} /$ day and $160 \mathrm{mg} /$ day in the acute exacerbation of schizophrenia and schizoaffective disorder: A 6-week placebo-controlled trial. Ziprasidone Study Group. Neuropsychopharmacology. 1999;20(5):491-505.

4. Keck PE Jr, Reeves KR, Harrigan EP. Ziprasidone in the short-term treatment of patients with schizoaffective disorder: Results from two double-blind, placebo-controlled, multicenter studies. J Clin Psychopharmacol. 2001;21(1):27-35.

5. Strom BL, Eng SM, Faich G, et al. Comparative mortality associated with ziprasidone and olanzapine in real-world use among 18,154 patients with schizophrenia: The Ziprasidone Observational Study of Cardiac Outcomes (ZODIAC). Am J Psychiatry. November 1, 2010. [Epub ahead of print].

6. Chouinard G, Jones B, Remington G, et al. A Canadian multicenter placebo-controlled study of fixed doses of risperidone and haloperidol in the treatment of chronic schizophrenic patients. JClin Psychopharmacol. 1993;13(1):25-40.

7. Marder SR, Meibach RC. Risperidone in the treatment of schizophrenia. Am J Psychiatry. 1994;151(6):825-835.

8. Peuskens J. Risperidone in the treatment of patients with chronic schizophrenia: A multi-national, multi-centre, double-blind, parallelgroup study versus haloperidol. Risperidone Study Group. Br J Psychiatry. 1995;166(6):712-726.

9. Janicak PG, Keck PE Jr, Davis JM, et al. A double-blind, randomized, prospective evaluation of the efficacy and safety of risperidone versus haloperidol in the treatment of schizoaffective disorder. $J$ Clin Psychopharmacol. 2001;21(4):360-368.

10. Csernansky JG, Mahmoud R, Brenner R. A comparison of risperidone and haloperidol for the prevention of relapse in patients with schizophrenia. N Engl J Med. 2002;346(1):16-22.

11. Tran PV, Hamilton SH, Kuntz AJ, et al. Double-blind comparison of olanzapine versus risperidone in the treatment of schizophrenia and other psychotic disorders. J Clin Psychopharmacol. 1997;17(5):407-418.

12. Conley RR, Mahmoud R. A randomized double-blind study of risperidone and olanzapine in the treatment of schizophrenia or schizoaffective disorder. Am J Psychiatry. 2001;158(5):765-774.

13. Lawson WB. Racial and ethnic factors in psychiatric research. Hosp Community Psychiatry. 1986;37(1):50-54.

14. Frackiewicz EJ, Sramek JJ, Herrera JM, Kurtz NM, Cutler NR. Ethnicity and antipsychotic response. Ann Pharmacother. 1997;31(11): 1360-1369.
15. Lin KM, Finder E. Neuroleptic dosage for Asians. Am J Psychiatry. 1983;140(4):490-491.

16. Subramaniam M, $\mathrm{Ng} \mathrm{C}$, Chong SA, et al. Metabolic differences between Asian and Caucasian patients on clozapine treatment. Hum Psychopharmacol. 2007;22(4):217-222.

17. Sramek JJ, Sayles MA, Simpson GM. Neuroleptic dosage for Asians: A failure to replicate. Am J Psychiatry. 1986;143(4):535-536.

18. Ruiz P, Varner RV, Small DR, Johnson BA. Ethnic differences in the neuroleptic treatment of schizophrenia. Psychiatr $Q .1999 ; 70(2)$ : $163-172$.

19. Chinese Society of Psychiatry. The Chinese Classification and Diagnostic Criteria of Mental Disorders. 3rd ed. Jinan, China: Shandong Science and Technology Press; 2001.

20. Simpson GM, Angus JW. A rating scale for extrapyramidal side effects. Acta Psychiatr Scand Suppl. 1970;212:11-19.

21. Manos N, Lavrentiadis G, Gkiouzepas J. Evaluation of the need for prophylactic antiparkinsonian medication in psychotic patients treated with neuroleptics. J Clin Psychiatry. 1986;47(3):114-116.

22. Pfizer. Geodon prescribing information. New York, NY: Pfizer Inc; 2006.

23. Zhang XY, Zhou DF, Cao LY, Zhang PY, Wu GY, Shen YC. Risperidone versus haloperidol in the treatment of acute exacerbations of chronic inpatients with schizophrenia: A randomized double-blind study. Int Clin Psychopharmacol. 2001;16(6):325-330.

24. Kongsakon R, Trinidad-Onate P, Chaudhry HR, et al. Asian outpatients with schizophrenia: A double-blind randomized comparison of quality of life and clinical outcomes for patients treated with olanzapine or haloperidol. J Med Assoc Thai. 2006;89(8):1157-1170.

25. Chan HY, Lin WW, Lin SK, et al. Efficacy and safety of aripiprazole in the acute treatment of schizophrenia in Chinese patients with risperidone as an active control: A randomized trial. J Clin Psychiatry. 2007;68(1): 29-36

26. Zink M, Kuwilsky A, Krumm B, Dressing H. Efficacy and tolerability of ziprasidone versus risperidone as augmentation in patients partially responsive to clozapine: A randomised controlled clinical trial. J Psychopharmacol. 2009;23(3):305-314.

27. Kuwilsky A, Krumm B, Englisch S, Dressing H, Zink M. Long-term efficacy and tolerability of clozapine combined with ziprasidone or risperidone. Pharmacopsychiatry. 2010;43(6):216-220.

28. Simpson GM, Glick ID, Weiden PJ, Romano SJ, Siu CO. Randomized, controlled, double-blind multicenter comparison of the efficacy and tolerability of ziprasidone and olanzapine in acutely ill inpatients with schizophrenia or schizoaffective disorder. Am J Psychiatry. 2004; 161(10):1837-1847.

29. Addington DE, Pantelis C, Dineen M, Benattia I, Romano SJ. Efficacy and tolerability of ziprasidone versus risperidone in patients with acute exacerbation of schizophrenia or schizoaffective disorder: An 8-week, double-blind, multicenter trial. J Clin Psychiatry. 2004;65(12): 1624-1633.

30. Goodnick PJ, Rodriguez L, Santana O. Antipsychotics: Impact on prolactin levels. Expert Opin Pharmacother. 2002;3(10):1381-1391.

31. Lieberman JA, Stroup TS, McEvoy JP, et al. Effectiveness of antipsychotic drugs in patients with chronic schizophrenia. $N$ Engl $J$ Med. 2005;353(12):1209-1223.
Neuropsychiatric Disease and Treatment

\section{Publish your work in this journal}

Neuropsychiatric Disease and Treatment is an international, peerreviewed journal of clinical therapeutics and pharmacology focusing on concise rapid reporting of clinical or pre-clinical studies on a range of neuropsychiatric and neurological disorders. This journal is indexed on PubMed Central, the 'PsycINFO' database and CAS, and is the official

\section{Dovepress}

journal of The International Neuropsychiatric Association (INA). The manuscript management system is completely online and includes a very quick and fair peer-review system, which is all easy to use. Visit http://www.dovepress.com/testimonials.php to read real quotes from published authors. 